\title{
A Critical Review on Morat With Special Reference to Ayurveda Classical Texts
}

\author{
Review Article
}

Nangare Ninad B1* ${ }^{*}$, Deshpande Manasi $\mathbf{M}^{2}$, Kurulkar Manisha $A^{3}$

\author{
1. Assistant Professor, 2. Professor and Head, \\ Department of Dravyaguna, Bharati Vidyapeeth (Deemed to be University) College of Ayurved, Pune. \\ 3. Professor and Head, Department of Ayurved Samhita and Siddhant, \\ Keshav Ayurveda Medical College, Aklera, Rajasthan.
}

\begin{abstract}
Morat is considered as a significant medicinal plant in the indigenous system of medicine [Ayurveda] as it has wide application as single drug. It is very effective in various Urinary disorders. It has been observed that several species of plants which are referred under one common name, making them more controversial. Documentation defects in manuscripts, same synonym but different plants, geographic variations are some of the reasons attributed to it. Due to different languages and morphological similarities between plants generates controversy. In India, there are many plants which use a common name Morat and creates controversy. Under the name of Morat, botanical identity such as Leea macrophylla Roxb., Saccharum officinarum L., Alangium salvifolium (L.f.)Wang. Marsdenia tenacissima Wight. \& Arn., Maerua arenaria Hook, Chonemorpha fragrans Moon are considered as they exhibit same medicinal properties. The purpose of this work is to review and differentiate the plants which are known as Morat in Ayurved classical texts.
\end{abstract}

Key Words: Morat, Controversial plants, Bruhatryee, Nighantu.

\section{Introduction}

Over 2500 species of medicinal plants are documented in the classical texts of Ayurved. Beside the usefulness of plants in various disorders, today we are using comparatively very smaller number of plants for various ailments, because many of them have not been identified properly. Moreover, the same synonyms may be given to more than one plant, causing confusion in identifying the genuine plant. This confusion is compounded by the lack of a technically precise description of the complete plant. As per the Ancient Indian Literature, Controversial plant or Sandigdha drayas is a term used for medicinal plants having notorious botanicals as sources. Quantum of information gained from Ayurvedic \& other Sanskrit literature revealed various incidences where one common name is used for two or more entirely different plant species in traditional system of Medicines.

Morat is one of the controversial plants useful in Mootrakruchchhra (Dysuria), Mootraghata, (Anuria), Ashmari (Urinary calculi), Cephalalgia, Gulma and internal abscesses. Since Samhita period, the plant is in ambiguity \& still not clearly identified botanically. To tackle controversy of Morat, here an

\section{* Corresponding Author:}

Nangare Ninad B

Assistant Professor, Department of Dravyaguna,

Bharati Vidyapeeth College of Ayurved,

(Deemed to be University),

Pune, India.

Email Id: ninadnangare@gmail.com attempt had been made to review various Ayurved classical texts, Samhita, Commentaries, Nighantu, Chikitsagranth.

\section{Materials and methods}

All available Vedic literature including Puran, Samhita [Ayurved classical texts bruhatryee and Laghutrayee], various treatises of samhita period and nighantu [Ayurved Materia Medica] were searched with the key words like Morat or its parayaya (synonyms) names. Textbooks of dravyaguna and various commentaries available were reviewed and analyzed using above mentioned keywords.

Literature about various plant species were compiled from different modern books, flora, journals, web based search engines like Google, Google scholar, PubMed, AYUSH portal etc. The obtained information about drug Morat \& it's noted synonyms from the classical has been analyzed regarding its controversial aspect and presented in a systemic manner.

\section{Observations}

\section{Historical perspective of Morat}

Morat is well known in Ayurveda. It is used since time immemorial to till date in various purposes. In vedic period [2500-300 BC] Morat is not mentioned. But it definitely seems to be known in "Garud Puran" by the name Morata. (1)

It is observed that major Ayurvedic classical texts Samhita Period [300BC-1300AD] describes the Morat in various classification as follow- 


\begin{tabular}{|c|c|c|c|c|}
\hline \multirow[b]{2}{*}{ Classical texts } & & & & \\
\hline & $\begin{array}{l}\text { Terminology } \\
\text { used }\end{array}$ & Varga [ class] & Uses & Reference \\
\hline \multirow{4}{*}{$\begin{array}{l}\text { Charak Samhita } \\
\text { (1000 B.C - } 4 \text { A.D.) } \\
\text { Sushrut Samhita } \\
\text { (1000 B.C - } 2 \text { A.D.) }\end{array}$} & Moratam & Goras Varga & - & $(2)$ \\
\hline & Morat & Madhuraskandha & - & (3) \\
\hline & Morat & $\begin{array}{l}\text { Varunadi Gana, } \\
\text { Veeratarvadi Gana }\end{array}$ & Ashmari [ Urinary calculi] & $(4)(5)$ \\
\hline & Kshiramorat & Madhura Varga & $\begin{array}{l}\text { Kitak Danshachikitsa } \\
\text { [Diseases due to stinger] }\end{array}$ & $(6)(7)$ \\
\hline \multirow{3}{*}{$\begin{array}{l}\text { Ashtang Sangraha } \\
\text { (6 } 6^{\text {th }} \text { Century AD), } \\
\text { Ashtang Hriday } \\
\text { ( } 7 \text { th Century AD), }\end{array}$} & Morat & $\begin{array}{l}\text { Varunadi Gana and } \\
\text { Veeratarvadi Gana. }\end{array}$ & & (8) \\
\hline & Morat & $\begin{array}{l}\text { Varunadi Gana, } \\
\text { Veeratarvadi Gana }\end{array}$ & $\begin{array}{l}\text { Pittajashmari Bhedan } \\
\text { [Urinary calculi due to Pitta, } \\
\text { penetrative action] }\end{array}$ & $(9)(10)$ \\
\hline & Kshiramorat & - & - & $(11)$ \\
\hline \multirow{2}{*}{$\begin{array}{l}\text { Kashyap Samhita } \\
\text { (600BC-7th Century } \\
\text { AD) }\end{array}$} & Morat & Trivrutta Yoga & $\begin{array}{l}\text { Morat is used along with } \\
\text { Murva root. }\end{array}$ & (12) \\
\hline & Kshiramorat & - & $\begin{array}{l}\text { Murtragraha [urinary } \\
\text { problems] }\end{array}$ & (13) \\
\hline \multirow{3}{*}{$\begin{array}{l}\text { Harit Samhita } \\
\left(10-12^{\text {th }} \text { century AD) }\right. \\
\text { Bhavaprakash Samhita } \\
\text { (16 } 6^{\text {th }} \text { Century AD), }\end{array}$} & Morata & - & $\begin{array}{l}\text { Ashmari rog chikitsa } \\
\text { Khadiradi Kvath }\end{array}$ & $(14)(15)$ \\
\hline & Morat & $\begin{array}{l}\text { Varunadi Gana, } \\
\text { Veeratarvadi Gana }\end{array}$ & Ashmari [ Urinary calculi] & $(16)$ \\
\hline & Moratam & & $\begin{array}{l}\text { Pittaj Masurika } \\
\text { [chicken pox] }\end{array}$ & $(17)$ \\
\hline
\end{tabular}

Morat name is mentioned by Bruhatryee, Kashyap, Harit \& Bhavaprakash Samhita, while Kshirmorat is introduced in Sushrut Samita, Ashtang Hriday \& Kashyap Samhita. There is no any reference found in Bhel \& Sharangadhar Samhita.

It is observed that different names are used for drug Morat by various commentators [Table 2] as follow-

Table 2 Opinion of different commentators about Morat

\begin{tabular}{|c|c|c|c|c|c|c|c|c|c|}
\hline \multirow{2}{*}{ Name } & \multicolumn{9}{|c|}{ Commentators } \\
\hline & Brihatryee & CK & DA & AR & HE & SH & GA & YO & JY \\
\hline Ankolpushpa & + & + & + & & & & & & \\
\hline Hastiparni & + & + & + & & & + & & & \\
\hline Ikshumul & + & + & + & & & & & & \\
\hline Kshirmorat & + & & + & + & & & & & \\
\hline Kshirmurva & + & & & & + & & & & \\
\hline Karnamorat & + & & & & & & + & & \\
\hline Murva & + & + & & + & & & & & + \\
\hline Piluparni & + & + & + & & & & & + & \\
\hline
\end{tabular}

(Abbreviation :- CK-Chakrapania 11th Cen. AD, DA-Dalhana 12th Cen AD, AR-Arundatta 12th $13^{\text {th }}$ Cen AD, HEHemadri 13th $-14^{\text {th }}$ Cen AD, GA- Gangadhar 19th Cen AD, SH-Shivadas Sen 19th Cen AD, YO-Yogendranath 20th Cen $A D$, JY- Jyotish Chandra Saraswati $20^{\text {th }}$ Cen AD)

\section{Compendia}

Morat is mentioned in Virataradi gana \& Varunadi gana used in Pittaj Ashamari by Vrund Madhav and Chakradatta (18)(19). Chakradatt also mentioned in Guduchyadi kashay for Masurika.

Vangsen and Bhaishjya Ratnavali used Morat in Bhadravahaghruta for Mutraghat, Kushadyaghruta \& virataradigana for Ashmari. As Moratu, mentioned in Masurika and as Kshirmorat in Madhuvarga. (20)(21)(22)(23)

Yogaratnakar has prescribed Morat in Rasnaputik tail \& Guduchyadi yoga for Vatvyadhi \& Masurika respectively. (24)(25)

\section{Nighantu [Materia medica]}

Nighantu means lexicon or dictionaries dealing with Indian material medica, have their origin in 8-10 A.D collection of words, synonyms and the names of the medicinal substance. It throws light on to the secret meanings of the terms denoting different synonyms of the drugs. The ancient nighnatus were actually like Kosha containing the synonyms of drugs. Later the drugs had given the description of properties, actions and their uses. All most all the nighantus have also described various synonyms, attributes of drug Morat [Table 3, 4]. 


\begin{tabular}{|c|c|c|c|c|}
\hline Nighantu & Terminology & Synonyms & Class & Reference \\
\hline \multirow{3}{*}{$\begin{array}{l}\text { Paryayratnamala Nighantu } \\
\text { (8th century } A D)\end{array}$} & Morat & Piluparni & - & (26) \\
\hline & Moratum & Ikshumool & As dugdhavikar & \\
\hline & Kshirmorat & $\begin{array}{l}\text { Shitadru, Sarala, } \\
\text { Salya, Kshiravan }\end{array}$ & - & \\
\hline \multirow{2}{*}{$\begin{array}{l}\text { Ashtang Nighantu } \\
\text { (9th century } A D)\end{array}$} & Murva & Morati, Kshirmorat & Patoladi Gana & $(27)$ \\
\hline & Morat & - & $\begin{array}{l}\text { Varunadi Gana, } \\
\text { Veeratarvadi Gana }\end{array}$ & \\
\hline $\begin{array}{l}\text { Dhanvantari Nighantu } \\
\left(10^{\text {th }}-13^{\text {th }} \text { century } A D\right)\end{array}$ & $\begin{array}{l}\text { Morat as } \\
\text { Murva-vishesh } \\
\text { [type of } \\
\text { murva] }\end{array}$ & $\begin{array}{l}\text { Pilupushpa, } \\
\text { Madhustrav, } \\
\text { Kshiramorat, Tejani } \\
\text { \& Dirghamoola }\end{array}$ & $\begin{array}{l}\text { Guduchyadi varga } \\
\text { Jwaraghna [useful in fever] } \\
\text { and Kaphapittahar [pacify } \\
\text { Kaphapitta], Useful in } \\
\text { Trushna [thirst], Daha } \\
\text { [burning sensation], Shwas } \\
\text { [dyspnea]. }\end{array}$ & $(28)$ \\
\hline \multirow{2}{*}{$\begin{array}{l}\text { Nighantushesh }\left(12^{\text {th }} \text { cent }\right. \\
\text { AD) } \\
\text { Sodhala Nighantu }\left(12^{\text {th }}\right. \\
\text { century AD) }\end{array}$} & Murva & Morata & Aushadhi varga & $(29)$ \\
\hline & Murva & Morat & Guduchyadi varga & $(30)$ \\
\hline $\begin{array}{l}\text { Sidhhamantra Nighantu } \\
\left.\text { (13 th } 14^{\text {th }} \text { century } A D\right)\end{array}$ & -- & Kshiramorata & Pittakaphaghna varga & $(31)$ \\
\hline $\begin{array}{l}\text { Hridaydipak Nighantu ( } 13^{\text {th }} \\
\text { - } 14^{\text {th }} \text { century AD) }\end{array}$ & Morat & Kshiramorat & Ekpad varga & $(32)$ \\
\hline \multirow{2}{*}{$\begin{array}{l}\text { Madanpal Nighantu }\left(14^{\text {th }}\right. \\
\text { century AD) } \\
\text { Saraswati Nighantu }\left(14^{\text {th }}\right. \\
\text { century AD) }\end{array}$} & Murva & $\begin{array}{l}\text { Morat } \\
\text { Morat }\end{array}$ & $\begin{array}{l}\text { Abhayadi varga } \\
\text { Panivavarga as Dugdhavikar }\end{array}$ & $(33)$ \\
\hline & Murva & Morata & Lata varg & $(34)$ \\
\hline \multirow[t]{2}{*}{$\begin{array}{l}\text { Raj Nighantu } \\
\text { (15 century AD) }\end{array}$} & Morat & $\begin{array}{l}\text { Kirnapushpa, } \\
\text { Pilupatra, } \\
\text { Madhustrava, } \\
\text { Ghanamoola, } \\
\text { Dirghamoola, } \\
\text { Kshiramorat }\end{array}$ & $\begin{array}{l}\text { Guduchyadi varga, } \\
\text { Pacify Pitta, used in Daha, } \\
\text { Jwara }\end{array}$ & $(35)$ \\
\hline & Moratakam & $\begin{array}{l}\text { synonym for } \\
\text { Ikshumool }\end{array}$ & -- & $(36)$ \\
\hline \multirow[t]{2}{*}{$\begin{array}{l}\text { Kaiyadev Nighantu }\left(15^{\text {th }}\right. \\
\text { century } A D)\end{array}$} & Morat & $\begin{array}{l}\text { Ghanamool, } \\
\text { Kirnapushpa, } \\
\text { Madhustrava, } \\
\text { Kshiramorat, } \\
\text { Hastiparni, } \\
\text { Piluparna }\end{array}$ & $\begin{array}{l}\text { Aushadi varga, } \\
\text { madhurarasatmak and } \\
\text { having action on Jvara, } \\
\text { Trushna, Kapha pitta dosha. }\end{array}$ & $(37)$ \\
\hline & Moratum & Ikshumul & -- & \\
\hline \multirow{2}{*}{$\begin{array}{l}\text { Bhavaprakasha Nighantu } \\
\left(16^{\text {th }} \text { century } A D\right)\end{array}$} & Murva & Morata & -- & $(38)$ \\
\hline & & Moratum & Dugdha varg & (39) \\
\hline $\begin{array}{l}\text { Shiva Kosha (1 } 17^{\text {th }} \text { Century } \\
\text { AD) }\end{array}$ & Kshiramorat & Murva bhed & -- & $(40)$ \\
\hline $\begin{array}{l}\text { Shaligram Nighantu (19th } \\
\text { Century } A D)\end{array}$ & Murva & Morat & Guduchyadi varga. & $(41)$ \\
\hline
\end{tabular}

Table 4 Morat \& its Synonyms in Nighantu

\begin{tabular}{|l|l|l|l|l|l|l|l|l|}
\hline Synonyms & S.N. & M.N. & D.N. & H.D.N. & K.N. & R.N. & S.K. & A.M. \\
\hline $\begin{array}{l}\text { Ankolpushpa } \\
\text { Badhamulak }\end{array}$ & & & & & + & & \\
\hline $\begin{array}{l}\text { Dirghamula } \\
\text { Ghanamula }\end{array}$ & + & + & + & + & + & + & \\
\hline
\end{tabular}




\begin{tabular}{|c|c|c|c|c|c|c|c|c|}
\hline Hastiparni & & & & & + & & & \\
\hline Kirnapushpa & & + & + & & + & + & & \\
\hline Kolahya & & & & & & & & + \\
\hline Kolpatri & & & & & & & & + \\
\hline Kolpushpi & + & & & & & & & + \\
\hline Kshirmorat & & + & + & + & + & + & + & \\
\hline Kundali & & & & & & & & + \\
\hline Madhustrava & & + & + & & + & + & & \\
\hline Madhumul & & + & & & & & & \\
\hline Pilupatra & + & + & & & + & + & & + \\
\hline Pilupushpa & & & + & & & & & \\
\hline Pischileshwar & & & & & & & & + \\
\hline Purusha & & + & & & + & + & & \\
\hline Swedana & & & & & + & & & \\
\hline Tejani & & & + & & & & & \\
\hline Vanamula & & & & & & & & + \\
\hline
\end{tabular}

(Abbreviation :- S.N.-Soushrut Nighantu, M.N.-Madanadi Nighantu, D.N.-Dhanvantari Nighantu, H.D.N.-Hruday Dipak, K.N.-Kaiyadev Nighantu, R.N.-Raj Nighantu, S.K.-Shivakosh, A.M. Abhidhanmanjiri)

Table showing Kshirmorat and Dirghamula synonyms are used maximum times. Morat is treated as synonym for other plants like Murva, Iskumul, Ankolpushpa \& Piluparni. Morata is used as synonym for Murva, at the same time Dhanvantari Nighantu, Raj Nighantu \& kaiyadev Nighantu, stated as synonym as well as a separate drug.

Table 5 Morat as synonym for Murva, Ikshumul, \& Piluparni

\begin{tabular}{|c|c|c|c|c|}
\hline Texts & Murva(Morata) & Iskhumul & Piluparni & Ankolpushpa \\
\hline Ashtang Nighantu & + & & & \\
\hline Paryayratnamala & & + & + & \\
\hline Dhanvantari Nighantu & + & & & \\
\hline Shabdachandrika & + & & & \\
\hline Nighantu Shesha & + & & & \\
\hline Shodhal Nighantu & + & & & \\
\hline Madanpal Nighantu & + & & & \\
\hline Raj Nighantu & + & + & & \\
\hline Kaiyadev Nighantu & & + & & \\
\hline Bhavaprakash Nighantu & + & & & \\
\hline Saraswati Nighantu & + & & & \\
\hline Shivakosh & & + & + & + \\
\hline Sidhhasar Nighantu & & & + & \\
\hline
\end{tabular}

\section{Attributes}

A drug performs certain local and general actions by its Rasa and Guna, and certain specific therapeutic actions its Vipak, Veerya and Prabhav. The Attributes (Rasapanchak) of Morat in various texts is described in table 6.

Table 6- Attributes of Morat
Attributes
C.S S.S
R. N.
\begin{tabular}{l|l|l|l|} 
K. N. & M.D.N. & A.M. & A.R.
\end{tabular}

\begin{tabular}{|c|c|c|c|c|c|c|c|c|}
\hline Guna [properties] & Guru [Heavy] & & & & $\checkmark$ & & & \\
\hline Rasa $[$ taste] & Madhur [Sweet] & $\checkmark$ & $\checkmark$ & $\checkmark$ & $\checkmark$ & $\checkmark$ & $\checkmark$ & \\
\hline & Tikta [Bitter] & & & & & & & $\checkmark$ \\
\hline & Kashaya [Astringent] & & & $\checkmark$ & & & & \\
\hline Vipak [ post digestive effect] & Madhur [Sweet] & & & & $\checkmark$ & & & \\
\hline Virya $[$ potency] & Sheet [Cold] & & & & $\checkmark$ & & & \\
\hline
\end{tabular}

(Abbreviation :- C.S.-Charak samhita, S.S.-Sushrut samhita, K.N.-Kaiyadev Nighantu, R.N.-Raj Nighantu, M.D.N.Madanadi nighantu, A.M. Abhidhanmanjiri. A.R.N. Abhidan Ratnamala)

\section{Plants used as Morat}

Though drug has been listed in the Samhita and Nighantu, it remains controversial because several plants are used and sold under the name of 'Morat' in different parts of country. About six different plant species were to be taken in the name of Morat by Ayurveda practitioners in different parts of the country. 


\begin{tabular}{|r|l|}
\hline No. & Name Family \\
\hline 1 & $\begin{array}{l}\text { Saccharum } \\
\text { officinarum L. } \\
\text { Gramineae }\end{array}$ \\
\hline 2 & $\begin{array}{l}\text { Root } \\
\text { Alangium } \\
\text { salvifolium } \\
\text { (L.f.)Wang. } \\
\text { Alangiaceae }\end{array}$ \\
\hline 3 & $\begin{array}{l}\text { Leea macrophylla } \\
\text { Roxb. ex Hornem. }\end{array}$ \\
\hline & Vitaceae \\
\hline 4 & $\begin{array}{l}\text { Marsdenia } \\
\text { tenacissima } \\
\text { Wight. \& Arn. } \\
\text { Asclepiadaceae } \\
\text { Root, Bark }\end{array}$ \\
\hline 5 & $\begin{array}{l}\text { Maerua arenaria } \\
\text { Hook } \\
\text { Capparidaceae } \\
\text { Root }\end{array}$ \\
\hline 6 & \begin{tabular}{l} 
Chonemorpha \\
fragrans Moon \\
Apocynaceae \\
\hline
\end{tabular} \\
\hline
\end{tabular}

Table 7 The plants considered as Morat are [42][43][44] [45]

\begin{tabular}{|l|l|l|}
$\begin{array}{l}\text { Macroscopic } \\
\text { characters }\end{array}$ & $\begin{array}{l}\text { Geographical } \\
\text { character }\end{array}$ & Uses \\
\hline A paracter & &
\end{tabular}

\section{A perennial grass/herb \\ Extensively} with slender culms of varying thickness \& colour

Small tree up to 10 meter high

Large herbaceous shrub, $30-90 \mathrm{~cm}$ in height with perennial tuberous root

Large stout, twining shrub,

Large woody climber, with thick rootstock and thick leaves, flowers strongly scented It giant stout climber with large, sweet scented, white flower cultivated in hotter

parts throughout

India

The drier parts of

India, in plains \&

foothills of southern

India

Throughout the hotter parts of India, Assam, Burma

Abundantly found in Bengal, Madhyapradesh, western ghats, Gujarat, eastern \& western Himalaya Punjab, Sind, Gujarat, Central \& southern India

Dense moist forest throughout India up to $1500 \mathrm{~m}$ altitude
Diuretic, demulcent, cooling \& useful in urinary disorders Ref

Diarrhea, paralysis, piles, vomiting

Astringent, anodyne,

styptic, antiseptic.

mucilaginous; applied to wounds and sores; used for ringworm, guineaworm Purgative, antispasmodic, mild CNS depressant; used in colic. Bark in lipid disorders, also in polyuria and hemorrhagic diseases.

Bleeding piles, as

alterative in fevers; as

atonic in muscular debility.

Diarrhea, fever, polyuria, anemia, boils, leprosy, eye diseases, vomiting, poisoning, intermittent fever, gynaecological disorders

\section{Conclusion}

Due to lack of scientific names in the original texts, different plants are known in different parts of the country under one name which makes the drug controversial. It is more difficult to identify the actual plant among the various varieties of Morat. So there is need to conduct the systemic study with the help of literature, pharmacognostical, pharmacological \& clinical study for confirmation of species.

In present paper different plants named as Morat in Ayurved classical texts have been reviewed. Many plants are known with the same name in different regions. Literature review suggested that Morat is different from Murva \& useful in Urine disorders. On the basis of synonyms \& description mentioned in Ayurved classical text, two plant namely Marsdenia tenacissima Wight. \& Chonemorpha fragrans Moon may be taken as Morat. But proper identification and standardization should be done to identify exact species as Morat for desired therapeutic effect as well as minimized adulteration and substitutes.

\section{References}

1. B hat a charya R. Garudpuran by Krushnadyayapayan Vyas. Purvakhanda 204/81.2nd edition. Varanasi, India; Chaukhamba Sanskrit Sansthan; 2005.247p.
2. Acharya YT. Charaka Samhita with Ayurvedadipika.Commentary by Chakraprani, Sutrasthan 27/234. Varanasi, India; Chaukhamba Surbharati Prakashan; 2011.166p.

3. Acharya Y T. Charaka Samhita with Ayurvedadipika.Commentary by Chakraprani, Vimansthan 08/139.Varanasi, India; Chaukhamba Surbharati Prakashan; 2011.284p.

4. Acharya YT. Sushruta Samhita with Nibandhasamgraha commentary by Dalhana. Sutrasthan 38/08, 10.Varanasi; Chaukhambha Orientalia; 1997.142p.

5. Acharya YT. Sushruta Samhita with Nibandhasamgraha commentary by Dalhana. Chikitsasthan 07/09.Varanasi; Chaukhambha Orientalia; 1997.41p.

6. Acharya YT. Sushruta Samhita with Nibandhasamgraha commentary by Dalhana. Sutrasthan 42/18Varanasi; Chaukhambha Orientalia; 1997.158p.

7. Acharya YT. Sushruta Samhita with Nibandhasamgraha commentary by Dalhana. Kalpasthan 08/139.Varanasi: Chaukhambha Orientalia; 1997.284p.

8. Sharma S. Ashtangasamgraha, with Shashilekha Sanskrit commentary by Indu. Sutrasthan 16/11, 13. 2nd edition Varanasi; Chaukhambha Sanskrit Series Office; 2008.133p. 
9. Kunte AM, Sastri KR. Ashtanghrudaya with Sarvangasundara of Arunadatta and Ayurvedarasayana of Hemadri. Sutrasthana 15/ 21, 24. Varanasi; Chaukhambha Surabharati Prakashana; 2010.236p.

10. Kunte AM, Sastri KR. Ashtanghrudaya with Sarvangasundara of Arunadatta and Ayurvedarasayana of Hemadri. Chikitsa sthan 11/23. Varanasi; Chaukhambha Surabharati Prakashana; 2010.674p.

11. Kunte AM, Sastri KR. Ashtanghrudaya with Sarvangasundara of Arunadatta and Ayurvedarasayana of Hemadri. Chikitsasthan 08/113.Varanasi; Chaukhambha Surabharati Prakashana; 2010.651p.

12. Jivaka V, Kashyapa Samhita with Vidyotini Hindi Commentry. Chikitsasthan 3/15. Varanasi; Chaukhambha Sanskrita Sansthana; 2012.98p.

13. Jivaka V, Kashyapa Samhita with Vidyotini Hindi Commentry. Khilasasthan 10/144. Varanasi; Chaukhambha Sanskrita Sansthana; 2012.301p.

14. Pandey J. Harita Samhita text with Nirmala Hindi commentary. Tritiyasthan $31 / 10$ 1st edition. Varanasi; Chaukhambha Vishwabharati; 2010.410p.

15. Pandey J. Harita Samhita text with Nirmala Hindi commentary. Tritiyasthan 39/37 1 st edition. Varanasi; Chaukhambha Vishwabharati; 2010.435p

16. Srikantha Murthy, Bhavaprakasa of Bhavamisra (Text, English K.R. Translation, notes, appendices and index) Vol-2(Madhya \& Uttara Khanda), Varanasi, India; Krishna Das Academy; 1998.471-472p.

17. Srikantha Murthy, Bhavaprakasa of Bhavamisra (Text, English K.R. Translation, notes, appendices and index) Vol-2(Madhya \& Uttara Khanda) Varanasi, India; Krishna Das Academy ;1998.645p.

18. Tiwari P. Vrundamadhava athava siddhayoga. Ashmari adhikar 34/05, 27, 15. 1st edition. Varanasi; Chaukhambha vishvabharati; 2007.213p.

19. Bajpeyee J. Chakradutta of Chakrapani, with Hindi commentary. Ashmari chikitsa /2,13,20 \& Masurika chikitsa/29. 4th edition. Bombay; Khmraj Shrikrishnadass; 2006.165-166p,233p.

20. Nirmal S. Vangasena Samhita with English translation. Vol. 1 Mutraghat adhikar/41.1st Edn. Varanasi; Chaukhambha Sanskrit Series Office; 2004.559p.

21. Nirmal S. Vangasena Samhita with English translation. Vol.1 Ashmari rogadhikar/ 18,22.1st edition. Varanasi: Chaukhambha Sanskrit Series Office; 2004.565p.

22. Nirmal S. Vangasena Samhita with English translation. Vol.2 Masurika rogadhikar/51, 61.1st edition. Varanasi; Chaukhambha Sanskrit Series Office; 2004.233p.

23. Mishra B. Bhaishajyaratnavali with 'Vidyotini' Hindi Commentary. 19th edition. Varanasi; $\mathrm{Chaukhmbha} \quad$ Prakashan ; 2008.199p,710p,713p,718p.

24. Shastri L. P. Yogaratnakar. Vatvyadhi chikitsa(Rasnaputika taila, Swedalepan vidhi). 4th edition, Varanasi; Choukhambha Sanskrit Sansthan; 1988.541p,543p.

25. Shastri, L. P. Yogaratnakar. Masurika chikitsa(Guduchyadi yoga). 4th edition, Varanasi; Choukhambha Sanskrit Sansthan; 1988.264p.

26. Madhavkara. Paryayaratnamala. (E - nighantu). Developed by NIIMH (National Institute of Indian Medical Heritage), Hyderabad for CCRAS (Central Council for Research in Ayurvedic Science) New Delhi: 2012. (Available from: http:// www.niimh.nic.in/ebooks/eNighantu.). dated 12-01-2020 time11:35 IST

27. Vahata. Astanga Nighantu. Varunadi gana, (E nighantu). Developed by NIIMH (National Institute of Indian Medical Heritage), Hyderabad for CCRAS (Central Council for Research in Ayurvedic Science) New Delhi: 2012. (Available from: http:// www.niimh.nic.in/ebooks/e-Nighantu.). Dated 14-01-2020 time10:35 IST

28. Diwedi BK. Editor. Dhanvanatari Nighantu, Guduchyadi Varga. Varanasi; Chaukhamba Krishnadas Academy;2008.18p.

29. Hemachandra Suri. Nighantushesha. (E - nighantu). Vriksha kanda. Developed by NIIMH (National Institute of Indian Medical Heritage), Hyderabad for CCRAS (Central Council for Research in Ayurvedic Science) New Delhi: 2012. (Available from: http://www.niimh.nic.in/ebooks/e-Nighantu.) Dated 09-02-2020 time11:35 IST

30. Shodhala. Shodhala Nighantu. . (E - nighantu). Guduchyadi Varga, . Developed by NIIMH (National Institute of Indian Medical Heritage), Hyderabad for CCRAS (Central Council for Research in Ayurvedic Science) New Delhi; 2012. (Available from: http://www.niimh.nic.in/ebooks/eNighantu.) Dated 08-02-2020 time 07:30 IST

31. Keshava, Siddhamantra, (commentary of Bopadeva), (E - nighantu). Pittakaphaghna Varga. Developed by NIIMH (National Institute of Indian Medical Heritage), Hyderabad for CCRAS (Central Council for Research in Ayurvedic Science) New Delhi: 2012. (Available from: http:// www.niimh.nic.in/ebooks/e-Nighantu.) Dated 06-02-2020 time10:35 IST

32. Bopadeva. Hridayadipaka Nighantu. Priyavat Sharma. Editor. ekpad Varga,. (Commentary of Bopadeva) Varanasi; Chaukhamba Amarbharti Prakashan; 1977.09p

33. Nripa Madanpala. Madanpala Nighantu. Hariharprasad Tripathi. Editior. Abhyadi varga, Chaukhamba Krishnadas Academy; Varanasi; 2009.40p,171p

34. Sarasvati Nighantutu. Lata varga. (E - nighantu). Pittakaphaghna Varga. Developed by NIIMH (National Institute of Indian Medical Heritage), Hyderabad for CCRAS (Central Council for Research in Ayurvedic Science) New Delhi: 2012. (Available from: http://www.niimh.nic.in/ebooks/eNighantu.). Dated 01-02-2020 time11:35 IST

35. Narahari. Raja Nighantu, Tripathi Indradeva. Editor. Guduchyadi varga. Varanasi; Chaukhamba Krishnadas Academy; 2010.46p. 
36. Tripathi Indradeva editor. Raja Nighanțu by Narahari. Paniyadi varga. Varanasi; Chaukhamba Krishnadas Academy; 2010.

37. Sharma Priyavata editor. Kaiyadeva Nighantu by Kaiyadeva. Oushadhi Varga. Varanasi; Chaukhamba Orientalia; 2006.147p.

38. Chunekar KC. editor. Bhavaprakasha Nighanțu by Bhavamishra. Bhava Prakasha Purva Khanda. Guduchyadi Varga. Varanasi; Chaukhamba Bharati Academy; 2013.419p.

39. Chunekar KC. editor. Bhavaprakasha Nighantu by Bhavamishra Bhava Prakasha Purva Khanda. Dugdha Varga. Varanasi; Chaukhamba Bharati Academy; 2013.763p.

40. Shivadatta. Shiva kosha (E.nighantu); Hyderabad, National institute of Indian medicinal heritage for CCRAS, 2012(Available from: http:// www.niimh.nic.in/ebooks/e-Nighantu.) Dated 16-02-2020 time11:35 IST

41. Shaligram. Shaligrama Nighantu. Guduchyadi varga. Mumbai; Khemaraja Shrikrishnadas Prakashana; 2011. 134p.

42. Singh Balawant. Glossary of Vegetable Drugs in Bruhattrayi, 2nd edition. Varanasi; Chaukhamba Amarabharati Prakashana; 1999.295p, 315-316p, $324 p$.

43. JLN Shastry. A Text book on Dravyaguna, Vol. 5, Edition 2001, Varanasi; Chaukambha Publications; 2001.250p.

44. P.V.Sharma. Dravyaguna vigyan Vol.5, Varanasi; Chaukhamba Bharati Academy; reprint 2001.229-233p.

45. Acharya Bapalal Vaidya. Some Controversial Drugs of Indian Medicine. Varanasi; Chaukhamba Press; 1982.191-192p

46. Anonymous. The Ayurvedic Pharmacopoeia of India. Part 1. 1st edition. Vol. 4. New Delhi; Government of India, Ministry of Health and Family Welfare, Department of Indian Systems of Medicine and Homoeopathy; 2004.33p.

47. Sharma P.C, Yelne M.B, and Dennis T.J. Database on Medicinal Plants Used in Ayurveda. Vol. IV. Central Council for Research in Ayurveda and Siddhha, Ministry of Health \& Family Welfare, Govt of India; 2001187-190p.

48. Kirtikar, KR and Basu BD. Indian Medicinal Plants, Vol. IV, 2nd Edition. Allahabad; Lalit Mohan Basu; 1935,2662-2664p.
49. Anonymous. The Ayurvedic Pharmacopoeia of India. Part 1. 1st edition. Vol. 5. New Delhi; Government of India, Ministry of Health and Family Welfare, Department of Indian Systems of Medicine and Homoeopathy; 2006.5-7p.

50. Sharma, P.C, Yelne M.B. and Dennis T.J. Database on Medicinal Plants Used in Ayurveda. Vol. VII. Central Council for Research in Ayurveda and Siddhha, Ministry of Health \& Family Welfare, Govt of India 2001.1-10p.

51. Kirtikar KR and Basu BD. Indian Medicinal Plants, Vol. II, 2nd edition. Allahabad; Lalit Mohan Basu Allahabad; 1935.1237p.

52. Kirtikar, KR and Basu BD. Indian Medicinal Plants, Vol. I, 2nd edition. Allahabad; Lalit Mohan Basu; $1935.617 \mathrm{p}$

53. Sarvade Dattatray \& Acharya Rabinarayan. Leea macrophylla Roxb. ex Hornem.: An ethnomedicinal, ethnic food, economical, and pharmacological update. International Journal of Green Pharmacy. March, 2019; 13. 13-20p.

54. Anonymous. The Ayurvedic Pharmacopoeia of India. Part 1. 1st edition. Vol. II. New Delhi; Government of India, Ministry of Health and Family Welfare, Department of Indian Systems of Medicine and Homoeopathy; 1999. 116p.

55. Sharma P.C, Yelne M.B. and Dennis T.J. Database on Medicinal Plants Used in Ayurveda. Vol. VIII. Central Council for Research in Ayurveda and Siddhha, Ministry of Health \& Family Welfare, Govt. of India;2001;272-281p

56. Kirtikar KR and Basu BD. Indian Medicinal Plants, Vol. I, 2nd edition. Allahabad; Lalit Mohan Basu; 1935.189-190p.

57. Bhalakiya Hetal laxmichand, Dr. Nainesh Rajendra Modi .A Comprehensive Review on Maerua Oblongifolia (Forsk.) A. Rich. International Journal of Research in Advent Technology (IJRAT). April, 2019; Vol.7, issue-4; 721-727p.

58. Anonymous. Indian Medicinal Plants a Compendium of 500 species. Vol. II. Arya Vaidya Sala. Madras; Orient longman Ltd.; 2002.67-69p.

59. Hooker, J.D. Flora of British India assisted by various botanists. Vol.III. England; Reeve \& Co. Ltd.; 1882.661p.

60. Khare C. P. Indian medicinal plants: an illustrated dictionary. Springer; 2007. 144p. 